\title{
Peixes encontrados nos mercados de Manaus
}

\author{
E. M. S. Honda (*) \\ Instituto Nacional de Pesquisas \\ da Amazônia
}

\section{SINOPSE}

Neste trabalho relacionamos os peixes utilizados comercialmente em Manaus, fornecendo seus nomes vulgares e cientificos, locais de captura e variação le preços.

\section{INTRODUÇÃO}

A grande variedade de espécies que constitui a ictiofauna da região amazônica, principalmente aquelas de interesse comercial, tem atraído a atenção de numerosos estudiosos não só de Ictiologia como também de Economia, Sociologia e Bioquímica. O pesquisador menos acostumado, ao tentar identificar cientificamente o seu material, defronta-se, muitas vezes, com a variedade de nomes vulgares dada a uma mesma espécie ou, o mesmo nome vulgar a espécies diferentes.

Goeldi (1898) foi, talvez, o primeiro a publicar uma lista de nomes vulgares de peixes amazônicos com os correspondentes nomes científicos. Mais recentes, as publicações de Meschkat (1961), Carvalho (1967), Marlier (1968) e Knöppel (1970) também apresentam as denominaçōes populares além das designações científicas de algumas espécies da Amazônia.

Neste trabalho relacionamos os nomes vulgares de peixes encontrados com maior freqüência nos mercados de Manaus, dando os seus correspondentes em sistemática.

\section{GENERALIDADES}

Os mercados de Manaus são abastecidos por peixes capturados principalmente nas re- giões do Purus e Solimões. Outras regiōes como Careiro, Paraná-da-Eva, Negro, Baixo Amazonas e Madeira contribuem em menor escala.

A captura é realizada indiscriminadamente durante o ano todo, sendo considerado como safra o período em que as águas dos rios estão baixas, quando entảo, a pesca torna-se mais fácil.

Para a comercialização o peixe é classificado, de acordo com a maior procura, em 4 categorias. Na categoria especial estão incluídos o tucunaré, a pescada, o acará-açu e o acará prata. São considerados de primeira classe os seguintes peixes: tambaqui, pirarucu, sardinha, matrinchão e pacu. Compõem a segunda classe : curimatã, aracu, jaraqui, aruanã, pirandirá e branquinha. Estão reunidos na terceira classe: apapá, arraia, caparari, surubim, cuiucuiu, tamoatá, dourado, jandiá, mandubé, mapará, piramutaba, piraíba, piranha, acari, piranambu e demais peixes que aparecem esporadicamente nos mercados.

O pirarucu e os peixes da classe especial são vendidos a quilo e, de acordo com a satra ou entressafra, o preço varia de CrS 5,00 a $\mathrm{Cr} \$ 8,00$. A sardinha, jaraqui, pacu e branquinha são vendidos por "enfiada", ou seja, 5 peixes preso por uma tira de jauari e, de acordo com a época, o preço pode variar de Cr\$ 1,00 a $\mathrm{Cr} \$ 6,00$. O tambaqui pode ser vendido inteiro ou aberto longitudinalmente em bandas, variando o preço de $\mathrm{Cr} \$ 6,00$ a $\mathrm{Cr} \$ 20,00$ por um tambaqui inteiro, dependendo ainda do tamanho do exemplar. O chamado peixe liso ou de couro não tem grande aceitação, e o preço, de modo geral, não ultrapassa a Cr\$ 2,00.

LISTA DOS NOMES VULGARES DE PEIXES COM SEUS CORRESPONDENTES EM SISTEMÁTICA

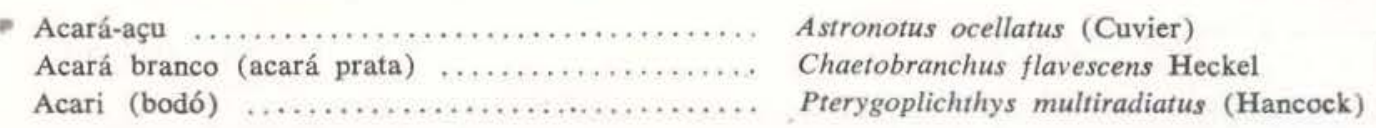

(*) - Bolsista do Conselho Nacional de Pesquisas. 


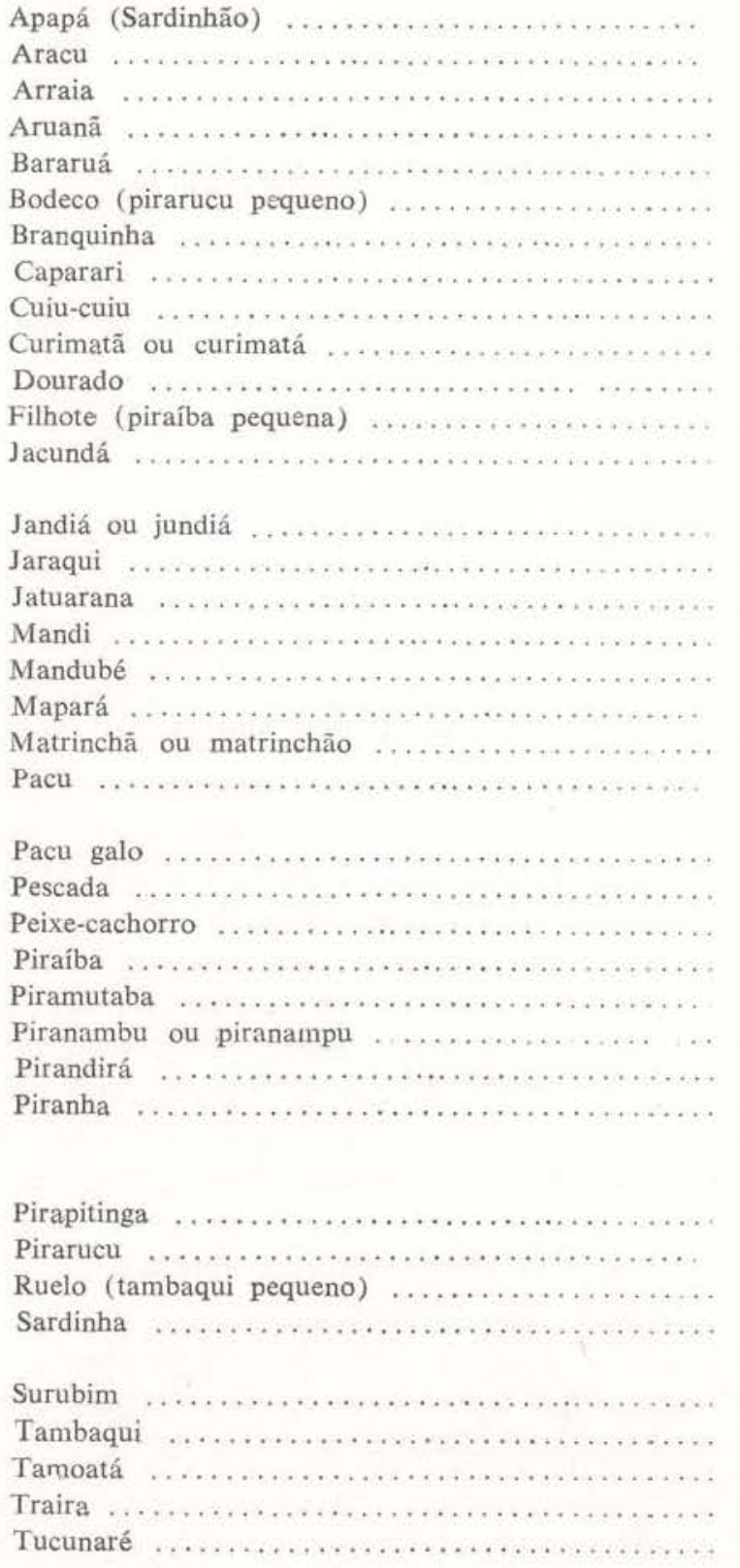

\section{SUMMARY}

This paper contains a list of scientific and vernacular names of the fishes found in the markets of Manaus.

\section{BIBLIOGRAFIA CITADA}

Carvalho, J. C. M.

1967 - A conservação da natureza e recursos naturais na Amazônia Brasileira. Atas do Simpósio sobre a Biota Amazônica, vol. 7 (Conservação da Natureza e Recursos Naturais) : 1-47.

Goeldi, E. A.

1898 - Primeira contribuição para o conhecimento dos peixes do valle do Amazonas e das Guyanas. Estudos ichthyologicos dos anos 1894-1898
Pellona flavipinnis (Valenciennes)

Leporinus fasciatus (Bloch)

Potamotrygon motoro Eigenmann \& Eigenmann

Osteoglossum bicirrhosum Vandelli

Uaru amphiacanthoides Heckel

Arapaima gigas (Cuvier)

Anodus laticeps (Valenciennes)

Pseudoplatystoma corruscans (Agassiz)

Oxydoras niger (Valenciennes)

Prochilodus nigricans Agassiz

Brachyplatystoma flavicans (Castelnau)

Brachyplatystoma filamentosum (Lichtenstein)

Crenicichl: saxatilis (Linnaeus)

Crenicichla strigata. Guenther

Rhandia schomburgkii Bleeker

Prochilodus insignis Schomburgk

Brycon hilarii (Valenciennes)

Pimelodella cristata (Mueller \& Troschel)

Ageneiosus ucayalensis. Castelnau

Hypophytalmus edentatus (Spix)

Brycon hilarii (Valenciennes)

Metynnis hypsauchen (Mueller \& Troschel)

Mylossoma duriventre (Cuvier)

Myloplus arnoldi Ahl

Plagioscion squamosissimus (Beckel)

Hydrolicus scomberoides (Cuvier)

Brachyplatystoma filamentosum (Lichtenstein)

Brachyplatystoma vaillantii (Valenciennes)

Pinirampus pinirampu (Spix)

Acestrorhynchus falcirostris (Cuvier)

Serrasalmus rhombeus (Linnaeus)

Pygocentrus nattereri Kner

Pygocentrus aureus (Spix)

Colossoma nigripinnis (Cope)

Arapaima gigas (Cuvier)

Colossoma bidens (Spix)

Triportheus elongatus (Giienther)

Triportheus angulatus (Spix)

Pseudoplatystoma fasciatum (Linnaeus)

Colossoma bidens (Spix)

Callichthys callichthys (Linnaeus)

Hoplias malabaricus (Bloch)

Cichla ocellaris Schneider

Cichla temensis Humboldt

KNOPPEL, H. A.

Bol. Mus. Paraense de Hist. Nat, e Ethnographia, II (1-4) + 443-488.

1970 - Food of Central Amazonian fishes. Contribution to the nutrient-Ecology of Amazonian rain-forest streams. Amazoniana, 2(3) : 257-353.

MARLIER, G,

1968 - Études sur les lacs de 1'Amazonic Centrale: II - Le plancton. III - Les poissons du lac Redondo et leur régime alimentaire; les chaines tropiques du lac Redondo; les poisson du rio Preto da Eva. Cadernos da Amazônia, Manaus. 11.: 1 -57.

Meschitat, A.

1251 - Fisheries of the Amazon Region. Report n? 1306. FAO. 